\title{
Endothelial Progenitor Cells $=\mathrm{EPC}=$ Elemental Pernicious Complexity
}

\author{
Ralf P. Brandes ${ }^{1}$ and Masuko Ushio-Fukai ${ }^{2}$
}

\begin{abstract}
Endothelial progenitor cells (EPCs) represent a heterogeneous population of cells with a pro-angiogenic potential that are derived not only from bone marrow but also from other tissues. Depending on the model and cell type used, the pro-angiogenic effect is a consequence of direct vascular integration, the paracrine release of growth factors and cytokines, or complex interactions with other cellular components like monocytes or platelets. The pro-angiogenic potential of EPCs is dependent on the particular type of EPC studied and modulated by the risk and life style factors of the patient as well as by local factors determining the homing to diseased tissue and the EPC proteome. In this Forum on EPCs these aspects will be covered in individual review articles, which are accompanied by two original research studies on the role of NADPH oxidases for EPC mobilization and the impact of organic nitrates on EPCs. Antioxid. Redox Signal. 15, 911-914.
\end{abstract}

C RCULATING ENDOTHELIAL PROGENITOR CELLS (EPCS) were first identified in adult peripheral blood by Asahara et al. in 1997 (4). Since then, extensive studies have been performed to understand the EPC phenotype, biology, and clinical potential. EPCs are mainly derived from adult bone marrow, but also from other tissues, and form a proangiogenic pool of cells. Neovascularization in the adult was initially thought to depend solely on angiogenesis, a process of new vessel formation via in situ proliferation and migration of pre-existing endothelial cells (12). However, accumulating evidence suggests that EPCs mobilized from the bone marrow and tissue reservoirs followed by their subsequent homing to diseased tissues also play an important role in physiological and pathological neovascularization $(1,3,23)$. EPCs participate in endothelial cell regeneration and neovascularization either in a direct or in an indirect way: They may directly differentiate into endothelial cells and incorporate into regenerating vasculature, a mechanism consistent with vasculogenesis, which has been described for embryonic vascularization. In addition, tissue-bound EPCs produce a variety of pro-angiogenic cytokines and growth factors, thereby promoting proliferation and migration of pre-existing endothelial cells to enhance angiogenesis. Thus, EPCs may contribute to vascular regeneration and neovascularization via activating not only vasculogenesis but also angiogenesis through paracrine functions. The discovery of EPC has opened up an opportunity of using these cells for autologous cardiovascular repair/regenerative therapies, as well as a disease biomarker. However, their therapeutic used has been severely limited by questions regarding their origin, cellular and molecular regulation, low homing efficiency to diseased tissues, the cellular heterogeneity of their population, and the lack of understanding of the in vivo functions of EPCs. These aspects shall be addressed in the current Forum, which consists of two original articles and eight reviews contributed by some of the leaders in the EPC field.

The term "EPC" in itself is an oversimplification, assembling an exceedingly heterogeneous cell population of different origin - not all EPC s derive from the bone marrowand, as judged on the basis of surface receptor expression, as well as of different functional nature. Moreover, depending on the culture condition used to transform blood- or bone marrow-derived cells into EPCs, different cell types are selected, expanded, or induced by means of differentiation, dedifferentiation, and transdifferenation. These global aspects, which are central to the field at large, are covered in basically all the articles. A separate article by Ergün et al. (9) will, however, explicitly cover tissue-resident stem cells of the vascular wall to emphasize the outstanding importance of this compartment for vascular regeneration. Indeed, resident vascular progenitor cells have several advantages over bone narrow-derived cells, as they are more committed toward vascular differentiation and differently respond to growth factors. Complex transplant experiments of differentially

\footnotetext{
${ }^{1}$ Institut für Kardiovaskuläre Physiologie, Fachbereich Medizin, Goethe-Universität, Frankfurt am Main, Germany.

${ }^{2}$ Department of Pharmacology, Center for Lung and Vascular Biology, Center for Cardiovascular Research, University of Illinois at Chicago, Chicago, Illinois.
} 
labeled cell population clearly demonstrated that vascular regeneration in part occurs through tissue-resident stem and progenitor cells. The number and regenerative capacity of these cells, however, over-exponentially decrease with age, and thus more work will be needed till ex vivo-expanded tissue-resident vascular cells will become available for therapeutic approaches.

Similar as for tissue-resident progenitor cells, also the number and biological activity of circulating EPCs of bone marrow-derived origin decline with age. Moreover, cardiovascular disease and systemic disorders such as diabetes have a strong negative impact on the regenerative capacity of EPCs, which is a major limiting factor for the success of autologous EPC transplantation. Assuming that the function of EPCs, at least in part, can be predicted on the basis of "-omics" analyses, Gremmels et al. (14) contributed a review on the transcriptome analysis of EPCs. This unbiased approach can be used to identify differences among EPC populations on the molecular level, far extending beyond the subjective characterization of cell population based on arbitrarily selected surface markers. Transcriptome analysis also helps to uncover the true changes occurring in EPCs during culture processes and most importantly to identify unexpected disease-specific alterations decreasing the function of EPCs.

In fact, although several complex interactions have already been identified to explain EPC dysfunction, truly novel mechanisms of dysfunction are still rare. Examples are specific dysfunctions of CXCR4 (29), altered p38 MAPK signaling (22), or unexpected regulations by microRNAs (10). Regarding redox regulation, EPCs appear to be surprisingly similar to endothelial cells although, quantitatively, their set of redoxactive enzymes such as nitric oxide (NO) synthases, NADPH oxidase $(20,26)$, and different forms of superoxide dismutases (7) differs from that of endothelial cells. The review by Fleissner and Thum (11), however, demonstrates that the fundamental principles of endothelial dysfunction can also be applied to EPCs: The function of EPC is dependent on NO and reactive oxygen species (ROS) (24). Although excess amounts of ROS, particularly superoxide, rapidly react with $\mathrm{NO}$ to reduce its bioactivity, ROS at physiological levels functions as signaling molecules to mediate various biological responses, including cell proliferation, migration, differentiation, and gene expression $(20,26)$. Besides mitochondria (8), NADPH oxidase is one of the major sources of ROS in endothelial cells (ECs) and stem/progenitor cells, and activated by various growth factors, cytokines, hypoxia, and ischemia. ROS derived from NADPH oxidase play an important role in redox signaling linked to angiogenesis (27) in ECs as well as stem/ progenitor cell mobilization, homing, and differentiation, thereby promoting neovascularization. This notion is important as it opens space for approaches to treat EPC dysfunction with similar tools as previously used to normalize endothelial function. This could be cardiovascular pharmacotherapy like the treatment with statins (28) or angiotensin II receptor blockers (17), but also live style interventions (18). Among these, particularly, physical exercise and training have beneficial effects on endothelial function via mobilization of EPCs from the bone marrow. In this Forum, Möbius-Winkler et al. address (16) the mechanism by which acute and long-term exercise training affects mobilization of EPCs. They suggest that acute exercise increases ROS generation via activation of NADPH oxidase, which stimulates EPCs' mobilization from the bone marrow, whereas chronic exercise training increases the bioavailable NO. These events are regulated by the antioxidant enzymes such as superoxide dismutases and glutathione peroxidase- 1 . Thus, effects of exercise training in regulating EPC function are mediated through alterations of the redox balance.

The family of ROS-producing NADPH oxidases consists of seven members (Nox1-Nox5 and DUOX1 and DUOX2). ECs express mainly Nox2 and Nox4, although recent findings suggest expression of Nox5 in human microvascular ECs. Most studies suggest that the classic NADPH oxidase Nox2 is involved in signaling activated by receptor tyrosine kinases such as vascular endothelial growth factor receptor and erythropoietin receptor. One of the original articles by Schroder et al. (21) provides the new information that hepatocyte growth factor-induced mobilization of EPCs in vivo and its pro-angiogenic effects in ECs are mediated through Nox2derived ROS. They also show that Nox2 activated by hepatocyte growth factor contributes to the phosphorylation of Jak2-STAT3 and Jnk, which mediate the pro-angiogenic response. As a possible downstream molecular targets of Jak2 and Jnk regulating EPCs mobilization, matrix metalloproteinases have been suggested, which may contribute to release the cells out of the bone marrow into the blood stream.

EPC numbers inversely correlate with cardiovascular disease risk $(15,28,30)$. Symptomatic coronary artery disease (CAD) is frequently treated with organic nitrates. In the other original article, Dr. Bauersachs and his group (25) compared the effects of the NO donors isosorbide dinitrate and pentaerythritol tetranitrate (PETN) on numbers and function of EPC and endothelial function in patients with symptomatic CAD. They show that PETN treatment increased EPC number and function by increasing antioxidative enzymes in ECs, whereas isosorbide dinitrate has no effects on EPC and rather worsens endothelial function in patients with CAD. This observation is important as it suggests that among a broad choice of organic nitrates, some might be more beneficial than others. Indeed, previous studies in animals and humans suggest that PETN differs from all other nitrates in that it does not induce nitrate tolerance and rather have additional antioxidative properties (13). The work by Thum et al. (25) in this Forum now demonstrates that this beneficial effect extends beyond a simple action on the vessel toward a systemic beneficial property, which also improves EPC function.

After the initial report on circulating endothelial precursors, a population of vascular smooth muscle progenitor cells was cultured from the circulating blood, adventitia, and bone marrow. During atherosclerotic plaque and/or neointima formation, neighboring cells such as mature endothelial cells and smooth muscle cells from the media migrate to the intima to replace dead endothelial cells through replication and acquisition of a synthetic, pro-proliferative phenotype. However, recent data suggest that vascular smooth muscle cells may also arise from EPCs and smooth muscle progenitors recruited from adventitia, resident vascular stem/progenitor cells, circulation, and other sites. Importantly, these cells have the potential to contribute to atherosclerotic plaque development and neointima formation. Thus, both endothelial and smooth muscle progenitors may act as a double-edged sword in the pathogenesis of arteriosclerosis. In this Forum, Campagnolo et al. (5) update the role of vascular progenitors in the development of arteriosclerosis, specially focusing on the nature, the characterization, the mechanisms of differentia- 
tion, and the controversial issues regarding the role of progenitor cells in cardiovascular disease progression. Understanding the mechanism for controlling fate decision of EPC differentiation into endothelial and smooth muscle is fundamental for both basic research and for the treatment of atherosclerosis.

A central factor limiting the success of the current therapeutic approaches of EPC therapy is the low homing efficiency of EPCs to diseased tissue, in particular if not inflamed. Homing of EPCs predominantly depends on the chemokines attracting these cells into the target tissue and on the receptors present on EPCs to allow an interaction with the target tissue. The review by Chavakis and Dimmeler (6) specifically addresses the complex process of EPCs homing, covering all important aspects from the attraction of the cells by chemokines, the rolling induced by selectins and the firm adhesion via integrins and adhesion molecules as well as the processes of diapedesis and tissue integration. This detailed article also analyzes the signal transduction of EPCs occurring during these processes and sheds light on the complex interactions between the signaling pathways and the differences in receptor expression and signaling between EPC populations.

The remaining article of this Forum by Asahara et al. (2) is devoted to therapeutic aspects of EPCs. It updates the potential value and current limitation of EPCs for therapeutic angiogenesis in ischemic diseases, covering all aspects from a proper definition of EPCs to the different therapeutic approaches.

Collectively, this Forum on EPCs draws a complex picture. The flawed terminology of the EPC field gave rise to a large body of in part contradictive literature. It is now obvious that a careful definition of the specific EPC in focus must accompany any study on these cells and that findings in one EPC population are not necessarily transferable to a different population. Thus, in the best of all meanings, also in this science, the model selected largely predicts the outcome of a study. Scientifically, these developments are positive as they have helped tremendously in improving our understanding on EPC biology. Clinically, however, it is becoming progressively clearer that the initial expectations in EPCs as a versatile, highly potent regenerative tool were more than exaggerated. In humans, bone marrow-derived cells and even EPC s can be used for regenerative cell therapy, but so far, the functional improvements elicited by this technically advanced and laborious approach, which is not without clinical risks, are relatively minor (19). The challenge of today's translational regenerative medicine therefore will be to select the best EPC population for an individual therapeutic intervention, and to expand these EPCs ex vivo to a significant number sufficient for in vivo approaches. Finally, it is essential to functionally improve these cells so that they have a higher homing and integration capacity and a strong functional impact.

\section{Acknowledgments}

This article was supported by grants from the Deutsche Forschungsgemeinschaft (SFB815/TP1 and SFB834/TPA2) and the excellence cluster cardio-pulmonary system to R.P.B., as well as National Institutes of Health R01 HL077524 and HL077524-S1, American Heart Association (AHA) GrantIn-Aid 0755805Z, and AHA National Center Research Program Innovative Research Grant $0970336 \mathrm{~N}$ to M.U.-F.

\section{References}

1. Aicher A, Zeiher AM, and Dimmeler S. Mobilizing endothelial progenitor cells. Hypertension 45: 321-325, 2005.

2. Alev C, Ii M, and Asahara T. Endothelial progenitor cells: a "novel" tool for the therapy of ischemic disease. Antioxid Redox Signal 15: 949-965, 2011.

3. Asahara T, Masuda H, Takahashi T, Kalka C, Pastore C, Silver M, Kearne $\mathrm{M}$, Magner $\mathrm{M}$ and Isner JM. Bone marrow origin of endothelial progenitor cells responsible for postnatal vasculogenesis in physiological and pathological neovascularization. Circ Res 85: 221-228, 1999.

4. Asahara T, Murohara T, Sullivan A, Silver M, van der Zee R, Li T, Witzenbichler B, Schatteman G, and Isner JM. Isolation of putative progenitor endothelial cells for angiogenesis. Science 275: 964-967, 1997.

5. Campagnolo P, Wong MM, and Xu Q. Progenitor cells in arteriosclerosis: good or bad guys? Antioxid Redox Signal 15: 1013-1027, 2011.

6. Chavakis E and Dimmeler S. Homing of progenitor cells to ischemic tissues. Antioxid Redox Signal 15: 967-980, 2011.

7. Dernbach E, Urbich C, Brandes RP, Hofmann WK, Zeiher $\mathrm{AM}$, and Dimmeler S. Antioxidative stress-associated genes in circulating progenitor cells: evidence for enhanced resistance against oxidative stress. Blood 104: 3591-3597, 2004.

8. Di S, V, Cencioni C, Zaccagnini G, Magenta A, Capogrossi MC, and Martelli F. p66ShcA modulates oxidative stress and survival of endothelial progenitor cells in response to high glucose. Cardiovasc Res 82: 421-429, 2009.

9. Ergün S, Tilki D, and Klein D. Vascular wall as a reservoir for different types of stem and progenitor cells. Antioxid Redox Signal 15: 981-995, 2011.

10. Fleissner F, Jazbutyte V, Fiedler J, Gupta SK, Yin X, Xu Q, Galuppo P, Kneitz S, Mayr M, Ertl G, Bauersachs J, and Thum T. Short communication: asymmetric dimethylarginine impairs angiogenic progenitor cell function in patients with coronary artery disease through a microRNA-21dependent mechanism. Circ Res 107: 138-143, 2010.

11. Fleissner $\mathrm{F}$ and Thum T. Critical role of the nitric oxide/ reactive oxygen species balance in endothelial progenitor dysfunction. Antioxid Redox Signal 15: 933-948, 2011.

12. Folkman J and Shing Y. Angiogenesis. J Biol Chem 267: 10931-10934, 1992.

13. Gori T and Daiber A. Non-hemodynamic effects of organic nitrates and the distinctive characteristics of pentaerithrityl tetranitrate. Am J Cardiovasc Drugs 9: 7-15, 2009.

14. Gremmels H, Fledderus JO, van Balkom BWM, and Verhaar MC. Transcriptome analysis in endothelial progenitor cell biology. Antioxid Redox Signal 15: 1029-1042, 2011.

15. Hill JM, Zalos G, Halcox JP, Schenke WH, Waclawiw MA, Quyyumi AA, and Finkel T. Circulating endothelial progenitor cells, vascular function, and cardiovascular risk. $N$ Engl J Med 348: 593-600, 2003.

16. Möbius-Winkler S, Schuler G, and Adams V. Endothelial progenitor cells and exercise-induced redox-regulation. $A n$ tioxid Redox Signal 15: 997-1011, 2011.

17. Muller P, Kazakov A, Jagoda P, Semenov A, Bohm M, and Laufs U. ACE inhibition promotes upregulation of endothelial progenitor cells and neoangiogenesis in cardiac pressure overload. Cardiovasc Res 83: 106-114, 2009.

18. Sandri M, Adams V, Gielen S, Linke A, Lenk K, Krankel N, Lenz D, Erbs S, Scheinert D, Mohr FW, Schuler G, and Hambrecht R. Effects of exercise and ischemia on mobilization and functional activation of blood-derived 
progenitor cells in patients with ischemic syndromes: results of 3 randomized studies. Circulation 111: 33913399, 2005.

19. Schachinger V, Erbs S, Elsasser A, Haberbosch W, Hambrecht R, Holschermann H, Yu J, Corti R, Mathey DG, Hamm CW, Suselbeck T, Assmus B, Tonn T, Dimmeler S, and Zeiher AM. Intracoronary bone marrow-derived progenitor cells in acute myocardial infarction. $N$ Engl J Med 355: 1210-1221, 2006.

20. Schroder K, Kohnen A, Aicher A, Liehn EA, Buchse T, Stein S, Weber C, Dimmeler S, and Brandes RP. NADPH oxidase Nox2 is required for hypoxia-induced mobilization of endothelial progenitor cells. Circ Res 105: 537-544, 2009.

21. Schröder K, Schütz S, Schlöffel I, Bätz S, Takac I, Weissmann N, Michaelis UR, Koyanagi M, and Brandes RP. Hepatocyte growth factor induces a proangiogenic phenotype and mobilizes endothelial progenitor cells by activating Nox2. Antioxid Redox Signal 15: 915-923, 2011.

22. Seeger FH, Haendeler J, Walter DH, Rochwalsky U, Reinhold J, Urbich C, Rossig L, Corbaz A, Chvatchko Y, Zeiher AM, and Dimmeler S. p38 mitogen-activated protein kinase downregulates endothelial progenitor cells. Circulation 111: 1184-1191, 2005.

23. Takahashi T, Kalka C, Masuda H, Chen D, Silver M, Kearney $M$, Magner $M$, Isner JM, and Asahara T. Ischemia- and cytokine-induced mobilization of bone marrow-derived endothelial progenitor cells for neovascularization. Nat Med 5: 434-438, 1999.

24. Thum T, Fraccarollo D, Schultheiss M, Froese S, Galuppo P, Widder JD, Tsikas D, Ertl G, and Bauersachs J. Endothelial nitric oxide synthase uncoupling impairs endothelial progenitor cell mobilization and function in diabetes. Diabetes 56: 666-674, 2007.

25. Thum T, Wiebking V, Ertl G, and Bauersachs J. Organic nitrates differentially modulate circulating endothelial progenitor cells and endothelial function in patients with symptomatic coronary artery disease. Antioxid Redox Signal 15: 925-931, 2011.

26. Urao N, Inomata H, Razvi M, Kim HW, Wary K, McKinney R, Fukai T, and Ushio-Fukai M. Role of nox2-based NADPH oxidase in bone marrow and progenitor cell function involved in neovascularization induced by hindlimb ischemia. Circ Res 103: 212-220, 2008.
27. Ushio-Fukai M, Tang Y, Fukai T, Dikalov SI, Ma Y, Fujimoto M, Quinn MT, Pagano PJ, Johnson C, and Alexander RW. Novel role of gp91(phox)-containing $\mathrm{NAD}(\mathrm{P}) \mathrm{H}$ oxidase in vascular endothelial growth factor-induced signaling and angiogenesis. Circ Res 91: 1160-1167, 2002.

28. Vasa M, Fichtlscherer S, Adler K, Aicher A, Martin H, Zeiher $\mathrm{AM}$, and Dimmeler S. Increase in circulating endothelial progenitor cells by statin therapy in patients with stable coronary artery disease. Circulation 103: 2885-2890, 2001.

29. Walter DH, Haendeler J, Reinhold J, Rochwalsky U, Seeger F, Honold J, Hoffmann J, Urbich C, Lehmann R, ArenzanaSeisdesdos F, Aicher A, Heeschen C, Fichtlscherer S, Zeiher AM, and Dimmeler S. Impaired CXCR4 signaling contributes to the reduced neovascularization capacity of endothelial progenitor cells from patients with coronary artery disease. Circ Res 97: 1142-1151, 2005.

30. Werner N, Kosiol S, Schiegl T, Ahlers P, Walenta K, Link A, Bohm M, and Nickenig G. Circulating endothelial progenitor cells and cardiovascular outcomes. N Engl J Med 353: 999-1007, 2005.

Address correspondence to: Prof. Ralf P. Brandes Institut für Kardiovaskuläre Physiologie Fachbereich Medizin Goethe-Universität Theodor-Stern-Kai 7, Frankfurt am Main 60596 Germany

E-mail: r.brandes@em.uni-frankfurt.de

Date of first submission to ARS Central, November 26, 2010; date of acceptance, December 2, 2010.

$\begin{aligned} & \text { Abbreviations Used } \\ \mathrm{CAD} & =\text { coronary artery disease } \\ \mathrm{EC} & =\text { endothelial cell } \\ \mathrm{EPC} & =\text { endothelial progenitor cell } \\ \mathrm{NO} & =\text { nitric oxide } \\ \mathrm{PETN} & =\text { pentaerythritol tetranitrate } \\ \mathrm{ROS} & =\text { reactive oxygen species }\end{aligned}$

